\title{
Intuiciones en el hacer, reflexiones acerca del quehacer cotidiano en la intervención comunitaria
}

Gutiérrez Monclus, Pamela Loreto

\begin{abstract}
- Resumen
El desempeño de Terapeutas Ocupacionales en el campo de la intervención comunitaria viene siendo desde hace años un área de trabajo y aporte desde nuestra profesión. A la luz de reflexiones en torno al Socioconstruccionismo, la Investigación Acción Participativa, la Educación Popular y las Perspectivas Feministas, este artículo busca hacer una reflexión a partir de la práctica, de algunos dilemas que nos encontramos en nuestro quehacer cotidiano.
\end{abstract}

Estas consideraciones surgen del trabajo profesional realizado durante cinco años en Atención Primaria en un equipo de salud mental y de las reflexiones que continuamente se realizaron grupalmente. De esta base, es de donde constituyo una reflexión de estas cuestiones a partir de ciertos parámetros teóricos, buscando problematizar aspectos como el uso de categorías monolíticas y unificadoras para entender a nuestro "sujeto" de intervención, así como, la forma de entender la noción de "identidad" desde una perspectiva narrativa y coherente. También me interesa adentrarme en aspectos en torno a la relación sujeto-objeto de la intervención y las implicancias ético-políticas de estos posicionamientos, como la necesaria reflexión respecto de la consideración como "expertos" de los profesionales.

\begin{abstract}
- Abstract
Community intervention has been for long a field of work for occupational therapists. On the light of reflections around socio-construccionism, Participative Action Research, popular education and feminist perspectives, the present article seeks to reflect, departing from practice, about some of the dilemmas we find in our everyday work.

The present reflections come from my professional practice in a primary health care unit where I worked for a period of five years. I was there as part of the mental health unit, and I gathered group reflections that constitute now a time and space in the reflection of these issues. Limited to my current position, I am seeking to problematize some aspects such as the use of monolithic and unifying categories in order to understand our "subject" of intervention, and, at the same time, the understanding of the notion of identity from a narrative and coherent perspective. Also I shall explore some aspects related to the subject-object relation in intervention and the ethic and political implications of those positionings, as a necessary reflection about professional expertness.
\end{abstract}

Terapeuta Ocupacional. Licenciada en Ciencias de la Ocupación. Diplomada en Salud Mental. Estudiante de Doctorado en Psicología Social en la Universidad Autónoma de Barcelona.

Contacto > > Mail: pamelagutierrezm@yahoo.es Dirección: Passeig Vall d'Hebron 62,5-4, escalera A, CP: 08023, Barcelona España. Fono: (93) 4340475 


\section{Descripción de una práctica profesional en intervención comunitaria}

A continuación hago una reflexión acerca del trabajo realizado en Santiago de Chile, en el marco de la Unidad de Salud Mental de un Consultorio $\left({ }^{(V)}\right.$, ubicado en la zona sur de la capital.

Cuando comencé a trabajar desempeñé un cargo con características administrativas y técnicas dentro del Departamento de Salud en la Municipalidad. Paralelamente, en la Unidad de Salud Mental del Consultorio trabajaban tres personas; una psicóloga , una asistente social en salud mental y una técnica social. Junto a ellas y otras personas -una psiquiatra, una terapeuta ocupacional, psicólogas, secretarias- se comenzó a dar luz a un nuevo centro de atención de salud mental, fuera de los límites del consultorio; para lo cual, se debía conseguir financiamiento por medio de proyectos sociales que nos permitieran tener los insumos para las actividades del centro. A este nuevo proyecto, se le llamó, Centro de Desarrollo Personal, Familiar y Comunitario.

En mi caso particular, fue todo un desafío comenzar un nuevo proyecto, ya que estaba recién egresada, y llevar a cabo ideas y sueños era un lujo, claro que estaban bastante lejos respecto de la gente a quienes iban dirigidos. En la fiebre de proyectos que se debieron hacer, se lograron dos iniciativas: uno se conformó por actividades para jóvenes y adolescentes: talleres de murgas (de expresión artístico - musical), panadería y diseño gráfico; y otro, de actividades físicas para personas de tercera edad. Después de concretar todo ello, y a los seis meses de funcionamiento, se nos comunicó que el Centro se debía cerrar por falta de fondos. A las personas del equipo se nos reubicó en los consultorios, otras fueron despedidas, mientras que otras decidieron irse voluntariamente. Personalmente me tocó de nuevo ser parte del Departamento de Salud (como era mi labor inicialmente), trabajando con los proyectos dentro de ese espacio. Con el paso del tiempo y ante los desencantos, solicité pasar a ser parte de la Unidad de Salud Mental, lo que significaba menor estabilidad laboral pero quizás mejores proyecciones de desarrollo profesional, ya que entendía que no tenía mucho sentido hacer proyectos por hacer, sin continuidad, sin equipo de apoyo, con decisiones pactadas en torno a los intereses políticos de quienes estaban de turno en el poder.

Así, se inició un trabajo silencioso para dar apoyo a la continuidad de los proyectos desde la Unidad de Salud Mental. Fue silencioso por varios aspectos: era una persona nueva en el equipo de la Unidad; no se conocía mi rol y significó una construcción en el "hacer" ya que el ser terapeuta ocupacional pertenece tradicionalmente a la intervención terciara; además debía lograr mi integración al equipo de salud del Consultorio, del cual la Unidad de Salud Mental era parte integrante.

Poco a poco, el equipo fue cambiando, y constituimos un equipo totalmente nuevo, sólo con un poco de la historia de lo que había pasado en la antigua unidad, gracias a las conversaciones que se sostuvieron y los recuerdos que se disponían. Habitualmente durante los tres años siguientes fuimos parte de esta unidad: dos psicólogas, yo como terapeuta ocupacional que me desempeñaba como jefa de la unidad y una secretaria (oficial administrativo). Pero la dinámica y el trabajo realizado llevo a que el equipo fuera apoyado por más de 40 voluntarias - monitoras quienes trabajaban en el programa de rehabilitación de alcohol y drogas, rehabilitación psiquiátrica, monitoras de Taichi y de gimnasia, grupos de encuentro de adulto mayores, etc.

IV Se anexa poema "La casa de los perros locos" que en otras palabras describe este apartado.

V Aún con las dificultades que implica hablar de una experiencia y mantener el anonimato de quienes forman parte, he decidido por respeto a las demás personas implicadas en estas reflexiones, mantener sólo referencias generales acerca del lugar desde donde desarrollo el presente artículo. 


\section{- Ideas que sustentaban la Unidad de Salud Mental}

Los lineamientos de la Unidad de Salud Mental del Consultorio, contenidos en la propuesta técnica del año 2000, fueron promover una acción clínico- comunitaria, basándonos en el desarrollo de tres ejes: el primero de ellos, tenía relación con una concepción del territorio, se deseaba conocer a quienes se estaba atendiendo, de qué lugar venían y con ello, poder focalizar acciones de prevención en la zona. Con este fin, estructuramos una base de datos con las personas que asistían a la unidad, que contenía sus datos personales y dirección para poder "georeferenciar" (VII) estos datos y así tener una visión territorial de nuestras acciones. Para lograrlo, se trabajó en coordinación con el departamento de sistemas de información geográfica del municipio, que confeccionó los mapas para poder planificar nuestra acción del año siguiente, asociándolos con los datos epidemiológicos nacionales y el censo comunal ${ }^{(1)}$.

Otros aspecto a considerar fue poder establecer un estrecho contacto con organizaciones comunitarias $^{\cdot(1)}$ Esto fue un trabajo que se dio poco a poco sobre la base de establecer relaciones de conocimiento mutuo, confianza y amistad con las integrantes de algunas Juntas de Vecinos, o con personas de la comunidad interesadas en algo en particular (como apoyar o formar un grupo de personas con discapacidad de su barrio, o formar grupos de adultos mayores o de gimnasia).

Finalmente, la tercera orientación importante de la Unidad fue fortalecer el equipo de salud mental, y fomentar la integración en el consultorio, promoviendo la capacitación y el desarrollo humano de cada una de las integrantes.

Las estrategias para la consecución de estos ejes de trabajo fueron:

1.- Fortalecimiento de redes

2.- Participación comunitaria

3.- Capacitación del equipo

4.- Prioridad programa de alcohol y drogas y de violencia intrafamiliar.

5.- Alumnos en práctica de terapia ocupacional y psicología con enfoque clínico comunitario

6.- Priorizar acciones de prevención y promoción de salud mental en sectores según georeferencia.

VII. Un sistema de Información Geográfica según la definición del Nacional Center of Geografic Information and Analysis es un "sistema de hardware, software y procedimientos elaborados para facilitar la obtención, gestión, manipulación, análisis, modelado, representación y salida de datos espacialmente referenciados, para resolver problemas complejos de planificación y gestión" (NCGEA: 1990, vol. I - p.13).De esta definición se puede considerar, esencialmente, al S.I.G como una tecnología aplicada a la resolución de problemas territoriales, aunque las áreas de uso práctico pueden ser muy variadas (Chorley: 1987) En otras palabras, un S.I.G es sensible de aplicarse en cualquier área que requiera del manejo de información espacial. http://www.datarq.fadu.uba.ar 


\section{Desde dónde se realizan las reflexiones}

Es de interés reflexionar a la luz de los lineamientos que nos sustentaban, cómo nuestra práctica se fue configurando y en lo que necesariamente seguirá cambiando; y cómo con la reflexión del equipo pudimos ir resolviendo o no, dilemas del quehacer cotidano. Ahora, luego de un par de años de distancia de estas experiencias, quiero dialogar con estos dilemas y cruzarlos con algunos otros debates y diálogos encontrados en mi formación actual, poder cruzar algunos puntos de encuentro y de disenso, asumiendo la responsabilidad de que éste es "una" de las múltiples lecturas de esta experiencia y que en este documento, pretendo presentar sólo un punto de vista en tránsito, como dice Haraway:

"El yo que conoce es parcial en todas sus facetas, nunca terminado, total, no se encuentra simplemente ahí y en estado original. Está siempre construido remendado de manera imperfecta y, por lo tanto, es capaz de unirse a otro, ver junto al otro sin pretender ser el otro". (2), pp.332)

Comentar, que algunos de mis diálogos con nuestros dilemas, vendrán de "la Educación Popular", que aboga por una pedagogía dialógica en la que el educador y el educando trabajan sobre las maneras de ver el mundo, como un compromiso con los oprimidos sobre la base de la problematización de situaciones cotidianas para ubicar su origen ideológico. ${ }^{(3)}$. Otra línea de diálogo será "la Investigación Acción" que se define como una "propuesta metodológica inserta en una estrategia de acción definida, que involucra a las beneficiarias de la misma en la producción de conocimientos. Persiguiendo la transformación social, vista como totalidad y que supone la necesaria articulación de la investigación, educación y acción."(4), pp.19). Y por último, los aportes de "Perspectivas Feministas", en donde el conocimiento es parcial, y situado ${ }^{(2)}$, y las interventoras crean su propia teoría inductivamente en relación al contexto y la práctica contextual y éstas se involucran en un proceso de descubrir con los clientes, creando juntos experiencias y condiciones frente a las diferentes situaciones. ${ }^{(5)}$.

\section{- Algunas intuiciones "}

\subsection{Las categorías/nombres/narrativas/identidades}

Se comenzará por comentar algunas intuiciones en el "hacer", relacionadas con aspectos de categorías diagnósticas, narrativas coherentes y conceptos de identidad. Como equipo en más de una ocasión se recibieron críticas por no realizar diagnósticos, en los informes dirigidos a la escuela, ya que se prefería describir la situación y proponer alternativas tanto a nivel familiar como a nivel escolar, lo que no calzaba con el marco del modelo médico en el que estábamos insertas.

Cuando se desarrolló en el Consultorio el plan de atención psiquiátrica, tuvimos asesoría especializada para atender las situaciones de personas que estaban más allá de nuestras posibilidades. Este aporte, el equipo lo valoraba pero nos volvía más a este ámbito que queríamos conocer pero no "sobreutilizar". A nuestro juicio, cuando establecemos una categoría diagnóstica establecemos a la vez una realidad, que necesariamente excluye otras posibles, y tales modos de exclusión son obtenidos por medio de una objetivación de los sujetos a través de clasificaciones científicas. ${ }^{(6)}$ Por ejemplo, en el Programa de Depresión, construíamos significados asociados a la patología, como parte de la identidad y como herramienta terapéutica para promover una narrativa de vida coherente, entonces, la depresión pasaba a ser parte de los significados personales y constitutivos de la persona, si ella se asumía depresiva, asumiría la medicación, la terapia, los grupos y la psicoeducación.

Parte de la intervención de este programa consistió en trabajar sobre la idea de un "self" unitario, que fuese coherente y que estableciera una trayectoria de vida hacia la consecución de unos 
objetivos personales. Es así cómo, quienes presentaban un self fragmentado se entendían como un desorden psiquiátrico del self, que podía despatologizarse por medio de la creación de una historia de vida coherente. () Esto se constituía como parte de las acciones cotidianas de patologización de las personas, ya que se continuaba privilegiando los ideales de unidad e inteligibilidad como propósito de vida, sugiriendo que la construcción "natural" del mito de la persona es hacia un ser conocedor y único.

Por otra parte, este entendimiento de la persona nos lleva a dialogar ahora, con una concepción de la mujer como "esencial", sin considerar, por ejemplo, posiciones teóricas que nos hablan acerca de la construcción social de la "subjetividad" y en donde cada localización individual particular está interceptada por múltiples discursos que construyen la identidad sexual, y que a la vez estos forman parte de relaciones de poder. ${ }^{(6)}$

Esta necesidad de construcción de narrativas que parecieran coherentes, también la podemos llevar a los procesos de participación comunitaria, ya que "se hace necesario, entonces, encontrar un sentido a los hechos, una coherencia, que los articule a pesar de ser contrarios". (4) (pp. 25-26). Pero ¿Cómo poder expresarnos no linealmente?, ya que se entiende que los procesos de investigación- acción y educación, a partir de la reflexión Freiriana se constituyen en momentos no necesariamente secuenciales o lineales, pero si indispensables y articulados. (4) Muchos de nuestros informes finales de proyectos, rescataron sólo los aspectos relevantes, secuenciales y ciertamente perdimos la posibilidad de comunicar mucha de la riqueza de las reflexiones y experiencias.

\subsection{Los profesionales/los no profesionales/ las otras/nosotras}

Otro aspecto, tiene que ver con la profesionalización de la salud mental, la cual se ha vuelto un espacio cada vez más, de manejo profesional "exclusivo". Muchas veces se había recurrido anteriormente a personas cercanas, afines a la religión o de buena capacidad de escucha, pero con el tiempo se ha vuelto un ámbito más reservado. Esto se vivió en un comienzo en la Unidad de Salud Mental, donde muchos otros profesionales se incapacitaban al tener a alguien en su oficina con alguna expresión de emociones. Fue un desafío intentar que se "normalizara la salud mental" y a su vez incluir a otros actores "no profesionalizados" en la labor.

Es así como, el abrir espacios a las trabajadoras socio-sanitarias, monitoras, etc., fue un trabajo diario. Dentro de los principios de la Investigación Acción Participativa (IAP), se considera que el conocimiento científico y el popular se articulan críticamente, en un tercer conocimiento nuevo y transformador ${ }^{(4)}$. Nuestro camino iba recogiendo algunos pasos en este andar, pero ciertamente nos restaba mucho por caminar.

El pasar desde la relación tradicional del sujeto-objeto entre la investigadora- educadora y los grupos populares, a una relación de sujeto-sujeto ${ }^{(4)}$, aún era parte de un recorrido que sólo comenzábamos, tal como se refleja en esta cita del Informe de evaluación del la Unidad de Salud Mental, del 2002:

"Los voluntarios y trabajadores socio sanitarios, alumnos de la escuela continua en dependencia, nos han dado más desafíos y esperanzas y necesariamente nos llevan a asesorarlos en nuevos desafíos. La autogestión en salud, y la participación en salud, nos está mostrando sus frutos, y nos permite cambiar de rol, de ejecutores a asesores de equipos de trabajo, para que las intervenciones en la comunidad sean más dinámicas y oportunas."

La relación sujeto-sujeto se fundamenta mediante lo que Freire llama principio de dialogicidad, entendida como un diálogo reflexivo-concientizador, crítico, democrático y antidogmático; de comunicación, que imprime un sello de horizontalidad a la relación investigadora-educadora con 
los grupos populares. ${ }^{(4)}$ (pp. 29) Nosotras comenzábamos a transformar nuestras relaciones hacia una horizontalidad, pero nos quedaba aún, mucho que recorrer. Quizás nuestro principal acercamiento en este andar, era compartir palco, (entre profesionales y no profesionales), en la escuela de formación continua de trabajadoras socio-sanitarias ${ }^{(\underline{X})}$, donde es de suma relevancia el valor de la experiencia.

\subsection{Cumplimientos/lealtades}

Otro aspecto a considerar en la intervención comunitaria tiene que ver con el compromiso éticopolítico que se estableció en el trabajo, algo que en la labor comunitaria es inevitable (y en cual no). Ya desde la opción de trabajar en este ámbito y también desde las propias relaciones cotidianas, se hace necesaria la reflexión acerca de las relaciones de poder en que se está inserta y cómo se puede caer en su reproducción.

Desde las prácticas, cada vez se hacía más necesario, poder generar las estrategias, planes y programas con la comunidad, pero a la vez, se era parte de un consultorio que pertenecía a la red de salud institucional. Entonces, muchas veces las formas de hacer y pensar del equipo, se veían contrastadas con las normas ministeriales y lo que es peor, con el cumplimiento de objetivos de gestión, que se traducían en la entrega de un dinero como "premio" (que incidía a la vez en la economía personal) por lo que embarcarse en una tarea irreflexiva de solucionar un problema con los ojos de la autoridad era la forma de cumplir estas metas colectivas, que necesariamente desplazaban las intuiciones de acciones más locales y territoriales.

Entonces ¿qué pasaba con las iniciativas que surgían por la demanda de la comunidad?. La intención del equipo fue un progresivo giro a la integración comunitaria, lejos aún de lo que seria la investigación acción participativa, "que parte de la realidad concreta de los propios participantes del proceso" (4), (pp.26). Ciertamente por formación o deformación previa, a algunas nos interesaba poder gestionar nuestro plan de salud con la comunidad, y en el consultorio se contaba con un equipo de la comunidad y realizaban acciones, pero algo ocurría en nuestro "dentro" institucional que no nos permitía ver de igual forma, por ejemplo en los Consejos Técnicos, tanto a los profesionales como a los no profesionales (no sólo los técnicos - administrativos sino a quienes no eran parte de la institución). Finalmente creo que muchas de estas gestiones de conocimientopoder conllevaban ciertamente formas de control por lo que la comunidad estaba lejos de una participación real y aún más lejos de una articulación concreta.

Así, algunas de las acciones de la Unidad se jugaban entre dos frentes: un cumplimiento de las normas ministeriales y la demanda creciente de acciones en conjunto con la comunidad. ¿Cómo responder a estas dos lealtades?. La Investigación Acción Participativa se considera como un proceso que combina la investigación científica y la acción política para transformar la realidad social y económica. (4, pp. 19-20) El octavo principio de la IAP ${ }^{(10)}$ considera que el "compromiso político e ideológico de la investigador/a-educador/a es con el sector popular y su causa" (4) (pp. 39).

\subsection{Los dentro - fuera/lo público-privado /los otras/nosotras}

Otra de las intuiciones del "hacer", era que se nos hacía cada vez más necesario reflexionar acerca de la distinción del "dentro" institucional versus el "fuera" o exterioridad. Mucho del giro en torno a trabajar más con la comunidad, que se intentaba realizar, pasaba necesariamente por difuminar los límites de lo dentro y fuera, de lo privado y lo público, y situarnos en la reflexión de nuestra concepción de personas con diferencias pero con capacidades de aportar a la co-construcción de un espacio- tiempo, sin sentirnos amenazadas en nuestro tan mal entendido "rol" profesional. Fueron nuestras relaciones y diálogos con estos otras/nosotras, que nos fueron transformando desde lo íntimo a lo público, considerando como telón de fondo que "las tecnologías de discursos y materialidades van moldeando lo que somos y las relaciones que se pueden establecer en contextos concretos" ()ㅡ, pp. 293). 


\subsection{Este "ser" necesario}

Pero esta transformación social de la realidad, de la IAP, "que busca comprender la situación concreta y objetiva de la dominación de clases y la percepción que de ella tienen las personas dominadas, para -junto con ellas- producir los conocimientos necesarios definidos para dirigir acciones de transformación de la realidad" (4) (pp. 20), deja algunas dudas ahora a la luz de la experiencia. Por ejemplo, existen cuestionamientos en torno a esta idea de profesional o investigadora que se sitúan como indispensables (ser necesario). También aparecen interrogantes frente a este proceso de compresión - concientización, "que implicaría que las personas con las que se trabaja deben "darse cuenta" de los elementos ideológicos que sirven de status quo para mantener la situación de asimetría presente en la sociedad". (ํ)

Por una parte el pretender que nosotras somos necesarias para estos procesos, desconoce la capacidad demostrada en el día a día de los agrupamientos sociales para gestionar y resolver sus propios problemas ${ }^{(11)}$, y como dice Montenegro, M., Pujol, J :

"Puede hacer(se) una fuerte crítica (a) la capacidad de las educadoras o agentes externas situarse en un metanivel desde la cual son capaces de develar los verdaderos orígenes que subyacen a la ideología propia de las oprimidos/as". Este acercamiento asume una postura representacionista del conocimiento, al asumir una realidad que es necesario develar y que por medio de esta acción se darán las bases para la transformación social. (8).

Por ello, el reflexionar acerca de esta supuesta "experticia" debe pasar a ser una necesidad cotidiana de nuestra acción, reconociendo nuestro propio proyecto político en nuestras intervenciones y respetando el de las personas con las que trabajamos. Como cita Montenegro, K.:

"Esta definición de roles, o división de saberes, trae consigo la construcción de identidades a partir de esta misma división, es decir; que unos son "expertos", mientras que los otros son "pobres" y "necesitados". El ser, es decir la esencia, de las personas intervenidas se fija a partir de un lugar de poder, mientras que la esencia de los profesionales o expertos se construye a partir de la misma fijación de la identidad de los otros. Spivack se pregunta ¿Qué diríamos si el margen está siendo constituido para complacer la conveniencia institucional del colonizador?." ${ }^{\text {(의 }}$

IX Personas que se capacitaban para realizar acciones en el área de rehabilitación en drogas y alcohol . web del programa ecológico multifamiliar: www.multifamiliar.cl 
- Conclusiones. ¿De qué otras formas poder actuar?

La Unidad de Salud Mental estaba inserta dentro de un marco de conocimiento objetivo y científico erigido como el "conocimiento verdadero" y sus conexiones hacia la participación eran pasos de un camino difícil de recorrer, ya que los criterios a través de los cuales se entendía y se actuaba "sobre la realidad" se basaba en parámetros normativos sobre cómo deben vivir las personas, y cuáles deben ser los recursos a los que tengan acceso, o cuáles deben ser sus prácticas de acuerdo a parámetros de lo que se considera como normal y anormal. ${ }^{(8)}$

Entonces siguiendo a Haraway :

"nuestro problema es como (...) lograr una práctica crítica capaz de reconocer nuestras "tecnologías semióticas" para lograr los significados y un compromiso que consiga versiones fidedignas de un mundo "real" que pueda ser parcialmente compartido y que sea favorable a proyectos globales de libertad finita, de abundancia material adecuada, de modesto significado en el sufrimiento y felicidad limitada. (2).

Entonces una alternativa de acción que surge son los llamados "conocimientos situados" y las "conexiones parciales", entre posiciones materiales y semióticas. La propuesta consiste en la conexión con otros seres e involucrarse con ellos en una conversación cuidadosa ${ }^{(8)}$, que nos permita conectar desde nuestras respectivas posiciones, reconociendo que pueden ser cambiantes. Todo esto admitiendo los aspectos de co-contrucción de materialidad y socialidad (11) presentes en nuestras relaciones cotidianas. Como terapeutas ocupacionales el mundo de lo relacional (ambiente) abarca tanto a objetos y personas, y por tanto, ambos deben ser parte de nuestra atención cuidadosa y respetuosa en la intervención comunitaria.

Si nos situamos desde una concepción de posiciones de "sujeto" de Laclau y Mouffe, se asume que, no hay sujetos que se constituyan de una sola vez, única (y) para siempre, sino que hay constantes procesos de definición y redefinición de posiciones que son precarias y contingentes (como opuesto a "necesarias") y que son entendidas como lugares desde los cuales se crean identificaciones y se actúa. $\stackrel{(10)}{ }$.

Entonces el tipo de intervención que provee de capacidad a la comunidad para "darse cuenta", se redefine desde una posición en cambio, desde un tránsito si se quiere, para que por medio de la articulación de algunos puntos nodales -que remite a la idea de red de significados y posiciones de sujetos-, se pueda articular en torno a fijaciones precarias y dinámicas, y emprender así cierta transformación social. $\stackrel{(10)}{ }$

Este tipo de acción buscaría generar y reinventar con cada nueva intervención-acción una manera de hacer basada en el tiempo-espacio contextual en que la relación dialógica es establecida, reconociendo nuestras limitaciones y estableciendo un "dialogo cuidadoso" de las relaciones de poder que nos cruzan como profesionales y en un compromiso político como interventoras de apoyo a las comunidades con las cuales trabajamos. 


\section{- Agradecimientos}

Agradezco los aportes a este trabajo que han significado las discusiones con las integrantes de los grupos: Fractalidades en Investigación Crítica (FIC) y Creacción, junto a mis compañeras del curso: Reflexiones en Torno a la Intervención Comunitaria cuya docente es Marisela Montenegro, en el marco del Doctorado en Psicología Social Crítica de la Universidad Autónoma de Barcelona, que han sido fundamentales en este trabajo.

Agradecimientos para Soledad Martinez Labrín ${ }^{(\Perp)}$, por su traducción y diálogos para la construcción de este artículo y a Caterine Galaz Valderrama ${ }^{(I I I)}$, por su dedicada corrección al presente texto.

II Magister y estudiante del Doctorado en Psicología Social de la Universidad Autónoma de Barcelona, UAB.

III Licenciada en Cs. De la Comunicación y estudiante del Doctorado en Educación de la Universidad Autónoma de Barcelona, UAB. 


\section{Referencias}

\section{Libros:}

(1) Levav, I. (1992) Temas de salud mental en la comunidad. Washington: Organización Panamericana de la Salud (OPS).

(2) Haraway, D. (1995) Conocimientos Situados: La cuestión científica en el feminismo y el privilegio de la perspectiva parcial, en Haraway, D. Ciencia, Cyborgs y Mujeres. Valencia: Ediciones Cátedra

(3) Montenegro, M. (2001) Análisis crítico de la investigación acción participativa desde el proceso desarrollado en el barrio "Niño Jesús" de Caracas. En Rodríguez, T., Montañés, M., Martín, P. (2001) Practicas locales de creatividad social, construyendo ciudadanía/ 2 (pp. 279-300) España: El Viejo Topo.

(4) Gabarrón, L., Hernández, L. (1994) Cuadernos Metodológicos, Investigación Participativa. Madrid: Centro de Investigaciones Sociológicas.

(5) Fook, J. (2000) Deconstructing and reconstructing professional expertise. En Fawcett, B., Featherstone, J. Fook, J, Rossitier, A. Practice and research in social work (pp. 104 - 119)

(6) Rossiter, A. (2000). The Postmodern Feminist Condition. En Fawcett, B., Featherstone, J. Fook, J, Rossitier, A. Practice and research in social work (pp. 2438) London: Routledge.

(7) De Peuter, J. (1998) The Dialogics of Narrative Identity. En Mayerfeld Bell y M. Gardiner (eds.) Bakhtin and the human sciencies. London: Sage (pp. 32-45.)

\section{Manuscritos}

(8) Montenegro, M., Pujol, J. (2001) Conocimiento situado: un forcejeo entre el relativismo y la necesidad de fundamentar la acción. (manuscrito)

(9) Montenegro, K (2004). El Capítulo de la Mirada, Epistemologías, Teorías, Reflexiones y Prácticas, Universidad Autónoma de Barcelona, 2004 (manuscrito)

(10) Balasch, M., León, A. y Montenegro, M. (2003) Posiciones de sujeto y articulaciones: una propuesta para la reflexión y la acción. Material UOC (manuscrito).

\section{Conferencias}

(11) Spink, P. (2004, Junio) Seminario: "Más allá de la Psicología Organizacional. Seminario Psicología Social Actual", Universidad Autónoma de Barcelona, Barcelona, España. 
- Anexo

En otras palabras....

\section{LA CASA DE LOS PERROS LOCOS}

Mayra M.

\section{PREFACIO}

"He pensado nombres de libros, aunque aún no los tenga listos y me gustaría que uno se llame "la casa de los perros locos" o algo por el estilo cuando deje de hacer frío cuando ilumine la luna cuando me sienta libre marcaré la hoja y ordenaré estas libres líneas del Don Dante antes las tendré, arrebatadas de aire y con mucha tierra, pero como aire puro que me renueva." (La Autora)

\section{CAPITULO I}

La casa de los perros locos

Se parece a un patio de atrás oscuro, Con luces rojas, Hay charcos y barro $Y$ de vez en cuando Brilla una piedra en tornasol color. Hay un rey de los perros locos

Que se sienta en silla ancha y mesa amplia

Y come solo, sólo palabras

Le adulan, lo maquillan

Hay cuadros de las otras piezas

Pintados como hermosos

Y él no sale

Los mira y los encuentra bellos.

El es Él, el importante

$Y$ tiene un sequito de perros de

Segunda categoría que le adulan y le trasladan

Con cortinas y pisadas

Y lo visitan las locas con minifalda

Para conversarle de algo, no importa qué.

(.... así se pasea, en el reino...)

Hay unas cañerías que salen de cada cuadro $Y$ se rumorea por ellas y se ve el futuro, Hay muchos brujos, brujos burocráticos 
Que señalan el destino venidero.

(Siempre más allá del tiempo imaginado)

También hay ampliaciones

Distribuidas por el reino

Para dar servicios al pueblo

Ciertamente que con su consentimiento.

También hay un flujo impresionante de gente

Navegando por la cañerías

Y siendo transportadas de una pieza a otra.

También hay los que se mimetizan y se vuelven tan silla, tan mesa

Que al mirar el cuadro el rey no repara y

No los cambian, no los comentan.

También hay cañerías que no conozco

Pisos con doble fondo

Y piezas de doble estándar

$Y$ con entrada secreta

Los niños del pueblo a veces pasan

Y se comen las zanahorias de las dependencias

O hacen sonar alguna alarma (en la misión del CEDE, por ejemplo)

Así es este reino

Dura cerca de cuatro años

Y se renueva el rey

A veces se retocan los cuadros

Algunos se aburren de los mismos

Pero les encanta el frío que pasa por las cañerías

Y siempre hay algo, algún comentario,

(luego el rey puede cambiar de casa

o pasa a ser perro de segunda categoría en alguna dependencia)

Y este ir y venir ocurre cada día

De 8:30 a 17:30

en cada casa de los perros locos

los viernes sólo hasta las 16:30.

$Y$ a veces los perros obreros se escapan

unos cuantos minutos municipales del reino

para pensar, que así al menos, pueden protestar. 